\title{
O uso de medicamentos antieméticos em oncologia pediátrica: revisão integrativa
}

\author{
The use of antiemetic drugs in pediatric oncology: integrative review
}

El uso de fármacos antieméticos en oncología pediátrica: revisión integradora

Inayane Loiola Lima1*, Carolina Heitmann Mares Azevedo Ribeiro², Ana Cristina Baetas Gonçalves².

\section{RESUMO}

Objetivo: Identificar os principais medicamentos antieméticos relacionados à oncologia pediátrica e propor, através da assistência farmacêutica, estratégias para a melhor terapia durante o tratamento quimioterápico, garantindo a proteção e recuperação dos pacientes. Métodos: Revisão integrativa, sem restrições quanto o desenho ou idioma do estudo, selecionando publicações dos últimos 5 anos, que incluíam apenas crianças de 0 à menores de 18 anos em tratamento quimioterápico. Resultados: São várias classes medicamentosas aplicáveis, chegando as seguintes terapias: Alto risco emético: antagonistas de serotonina (Granisetrona, Ondansetrona ou Palonosetrona), Dexametasona e Aprepitanto. Moderado: antagonista de serotonina e Dexametasona. Baixo: antagonista de serotonina. Alguns desses medicamentos apresentam grande impacto financeiro, já que não compõem a padronização do Sistema Único de Saúde. Considerações finais: Na construção desta revisão integrativa, observa-se a importância da temática abordada, estando no foco de centros de referência na Europa e América do Norte, que recomendam a utilização dos antieméticos através de protocolos, utilizando classes medicamentosas seguras para essa faixa etária. Porém, ainda são poucos os estudos fora desses centros de referência, como no Brasil e América Latina, onde não foram encontrados estudos. Diante disso, é importante trazer e aplicar essa informação na nossa realidade, visando à melhoria da assistência prestada ao paciente.

Palavras-chave: Êmese, Oncologia, Pediatria, Assistência farmacêutica.

\begin{abstract}
Objective: Identify the main antiemetic drugs related to pediatric oncology and propose, through pharmaceutical assistance, strategies for the best therapy during chemotherapy treatment, ensuring the protection and recovery of patients. Methods: Integrative review, with no restrictions on the design or language of the study, selecting publications from the last 5 years, which included only children aged 0 to under 18 years undergoing chemotherapy. Results: There are several drug classes applicable, reaching the following therapies: High emetic risk: serotonin antagonists (Granisetron, Ondansetron or Palonosetron), Dexamethasone and Aprepitant. Moderate: Serotonin antagonist and Dexamethasone. Low: serotonin antagonist. Some of these drugs have a large financial impact, as they are not part of the standardization of the Unified Health System. Final considerations: The construction of this integrative review, the importance of the addressed theme is observed, being in the focus of reference centers in Europe and North America, which recommend the use of antiemetics through protocols, using safe drug classes for this age group. However, there are still few studies outside these reference centers, such as in Brazil and Latin America, where no studies were found. Therefore, it is important to bring and apply this information in our reality, aiming to improve the care provided to the patient.
\end{abstract}

Key words: Emesis, Oncology, Pediatrics, Pharmaceutical services.

${ }^{1}$ Hospital Oncológico Inf antil Octavio Lobo (HOIOL), Belém - PA. *E-mail: inayanelima@hotmail.com

2 Universidade Federal do Pará (UFPA), Belém - PA.

SUBMETIDO EM: 11/2021 


\section{RESUMEN}

Objetivo: Identificar los principales fármacos antieméticos para la oncología pediátrica y proponer, en la asistencia farmacéutica, estrategias para mejor terapia durante la quimioterapia, asegurando la protección y recuperación de los pacientes. Métodos: Revisión integradora, sin restricciones en el diseño o lenguaje del estudio, con publicaciones de los últimos 5 años, que incluyó niños de 0 a 18 años en quimioterapia. Resultados: Existen varias clases de fármacos, alcanzando las siguientes terapias: Alto riesgo emético: antagonistas de la serotonina (Granisetron, Ondansetron o Palonosetron), Dexametasona y Aprepitant. Moderado: antagonista de la serotonina y dexametasona. Bajo: antagonista de la serotonina. Algunos de estos fármacos tienen un gran impacto económico, ya que no integran el Sistema Único de Salud. Consideraciones finales: La importancia del tema abordado en el foco de centros de referencia en Europa y Norteamérica, que recomiendan el uso de antieméticos a través de protocolos, utilizando clases de medicamentos seguros para este grupo de edad. Sin embargo, aún existen pocos estudios fuera de estos centros de referencia, como Brasil y América Latina, donde no se encontraron estudios. Poreso, es importante acercar y aplicar esta información en realidad, con el objetivo de mejorar la atención al paciente.

Palabras clave: Emesis, Oncología, Pediatría, Atención farmacéutica.

\section{INTRODUÇÃO}

O câncer que acomete a criança e o adolescente (entre 0 e 19 anos), corresponde a várias doenças que exibem, como principal característica em comum entre elas, a multiplicação desordenada de células anormais, podendo ocorrer por todo organismo, expressando características próprias em relação à histopatologia, ao comportamento clínico e ao período de latência menor, crescendo e tornando-se bastante invasivo, af etando principalmente, as células do sistema sanguíneo e os tecidos de sustentação, esses devem ser estudados separadamente devido tais características, e por responder melhor à quimioterapia em razão do pred omínio da natureza embrionária dessas células (INSTITUTO NACIONAL DE CÂNCER JOSÉ ALENCAR GOMES DA SILVA (INCA), 2016; INCA, 2019; MUTTI CF, et al., 2018).

Diferentemente da maioria dos cânceres de adultos, que os fatores de risco estão associados com o estilo de vida, para as crianças e adolescentes, raramente os cânceres têm relação com modificações genéticas tornando-as propensas a apresentar um determinado tipo de câncer. Contudo, a exposição a fatores ambientais, como a radiação e/ou tabagismo passivo, podem elevar o risco da criança para alguns tipos de câncer; porém, para melhor compreensão do processo de causa e ef eito, é imprescindível o aumento do número de estudos (INCA, 2019).

Vale ressaltar que, está aumentando a ocorrência mundial de câncer infantil. Foram estimados 300 mil casos novos no mundo, em 2014, entre crianças e adolescentes de até 19 anos (INCA, 2019). A incidência nos países em desenvolvimento, onde a população de crianças chega a $50 \%$, essa proporção do câncer infantil chega até $10 \%$ do total de neoplasias. Já em países desenvolvidos, essa proporção diminui próximo de $1 \%$ (INCA, 2016).

Também cabe destacar, que os tipos predominantes de cânceres pediátricos (entre 0 a 19 anos) em todo o mundo são leucemia (28\%), sistema nervoso central (26\%) e linfomas (8\%) (MINISTÉRIO DA SAÚDE, 2017; INCA, 2019; MUTTI CF, et al., 2018). Os tumores ósseos, como o osteossarcoma e tumores da família Ewing, tem maior incidência em adolescentes e representam $5 \%$ dos tumores nessa faixa etária. Já a incidência do tumor ocular, retinoblastoma, compreende, aproximadamente, a 4\% dos tumores malignos pediátricos e raramente ocorrem em outra faixa etária (INCA, 2016; MINISTÉRIO DA SAÚDE, 2017).

O número de óbitos por câncer, que acometem crianças e adolescentes no Brasil, correspondem à segunda causa de morte, ocorrendo em 2017, para o sexo masculino, 1.467 e para o sexo feminino 1.086 casos (INCA, 2016; INCA, 2019). A faixa etária de 15 a 19 anos apresentou a maior incidência de morte no país (54,01 por milhão) e a faixa etária de menor risco foi encontrada no grupo entre 5 e 9 anos (INCA, 2016; MUTTI CF, et al., 2018). 
No que se refere à estimativa de sobrevida, independentemente do tipo de neoplasia em crianças e adolescentes (de 0 a 19 anos), o cálculo e de aproximadamente $64 \%$, variando entre as regiões, com $50 \%$ na Região Norte, 60\% na Região Nordeste, 65\% na Região Centro-Oeste, 70\% na Região Sudeste, e 75\% na Região Sul (INCA, 2016).

Essas informações são fornecidas pelos Centros de Registros de Câncer de Base Populacional (RCBP) ou de base hospitalar (RHC), que estão distribuídos pelo Brasil, que tem como principal objetivo, avaliar o impacto do câncer em uma determinada população. Porém, esses dados sofrem a influência da subnotificação e subdiagnóstico, já que os pacientes buscam atendimento em outros estados devido às dificuldades de acesso aos serviços especializados em determinadas regiões. Assim, novas estratégias estão sendo aplicadas para diminuir tais situações (INCA, 2016).

A evolução no tratamento do câncer na infância e na adolescência foi extremamente significativa nos últimos 40 anos, chegando a aproximadamente $80 \%$ de curas e diagnosticados precocemente e tratados em tempo oportuno nos centros especializados, sem deixar maiores sequelas na qualidade de vida após finalizar o tratamento adequado (INCA, 2019).

A modalidade terapêutica mais utilizada no tratamento do câncer é a quimioterapia, que utiliza combinação de um ou mais antineoplásicos, administrados em intervalos de tempo regulares, variando de acordo com o esquema terapêutico utilizado por cada paciente (SANTOS SLF, et al., 2015; INCA, 2019).

As consequências desse tratamento são toxicidades hematológicas e gastrointestinais, que levam às náuseas e à êmese induzidos por quimioterapia, os quais são os sintomas de maior ocorrência e, clinicamente, importantes quando relacionados ao tratamento para crianças e adolescentes com câncer, pois são responsáveis por perdas significativas na qualidade de vida geral e redução da adesão ao tratamento (ALMEIDA RGL, et al., 2015; MORA J, et al., 2019; MUTTI CF, et al., 2018; SANTOS SLF, et al.; 2015; SING EPC, et al., 2019).

As náuseas e a êmese, resultantes da quimioterapia que envolvem estímulos em áreas do sistema nervoso central e periférico através de vários tipos de receptores, destacando a dopamina (D2), serotonina (5HT-3) e neurocinina-1 (NK-1) por três diferentes vias: 1) agindo diretamente no centro do vômito, situado na medula espinhal ou indiretamente; 2) por ativação da zona de gatilho quimiorreceptora (do inglês chemo receptor trigger zone - CTZ); ou 3) pelos estímulos aferentes vagais originados pela liberação da serotonina após lesionar as células do intestino delgado (células enterocromafins) (ALMEIDA RGL, et al., 2015).

No que tange as náuseas, essas são definidas como uma sensação desagradável da necessidade de vomitar, frequentemente seguida de sintomas autonômicos, como: sudorese fria, hipotonia gástrica, refluxo do conteúdo intestinal para o estômago, entre outros. Enquanto o vômito ou êmese caracteriza-se pela expulsão rápida e forçada do conteúdo gástrico por meio da boca, causada por forte e sustentada contração da musculatura torácica e abdominal (QUEIROGA HM, et al., 2017).

Tais sintomas estão entre os ef eitos mais desagradáveis e frequentes da quimioterapia antineoplásica, e af etam cerca de $80 \%$ dos pacientes que não realizam profilaxia antiemética. Esses são classificados em agudos, quando os sintomas aparecem com menos de 24 horas do início do uso dos quimioterápicos, relacionados ao nível emetogênicos desses medicamentos. Ao passo que, para os sintomas após as 24 horas, sua classificação será tardia ou ainda, antecipatória, relacionados ao controle ineficaz das náuseas e vômitos agudos do ciclo atual ou anteriores (ALMEIDA RGL, et al., 2015).

Diante dos expostos, foram utilizadas as evidências e diretrizes publicadas por centros de referência internacionais, chamados de guias de prática clínica ou guidelines, que auxiliam os profissionais de saúde em suas decisões farmacoterapêuticas publicando orientações em relação ao manejo adequado das náuseas e vômitos induzidos pela quimioterapia, possibilitando assim, uma resposta apropriada ao tratamento quimioterápico (SANTOS SLF, et al., 2015; SING EPC, et al., 2019).

Desse modo, o objetivo desse trabalho foi revisar a literatura existente para identificar os principais medicamentos antieméticos relacionados à oncologia pediátrica, e através dos cuidados e da assistência 
farmacêutica, garantir a proteção e a recuperação dos pacientes, assegurando a melhor terapia de maneira racional e integral em todas as etapas do tratamento quimioterápico de acordo com Conselho Federal de Farmácia (CFF) (2016).

\section{MÉTODOS}

Trata-se de uma revisão integrativa da literatura em que os seguintes bancos de dados foram pesquisados: Medical Literature Analysis and Retrievel System Online (Medline), Literatura Latino Americana e do Caribe em Ciências da Saúde(Lilacs), Public/Publisher MEDLINE (Pubmed) e Cochrane Collaboration (Cochrane), além de estudos de centros de referências da área oncológica, como: American Society of Clinical Oncology (ASCO), European Society for Medical Oncology (ESMO), Multinational Association of Supportive Care in Cancer (MASCC), International Society of Pediatric Oncology (SIOP), Pediatric Blood e Cancer, Journal of Pediatric Hematology/Oncology e British Pharmacological Society.

Todas as bases foram consultadas no período de novembro de 2019 a abril de 2020, selecionando as publicações dos últimos 5 anos, realizadas com humanos, utilizando as seguintes palavras-chave em inglês: Emesis, oncology e pediatrics. Não houve restrição quanto ao desenho do estudo ou ao idioma da publicação, incluindo estudos apenas com crianças de 0 à menores de 18 anos. Para estudos com participação de adultos e crianças, descreveu os resultados pediátricos separadamente ou a média de idade dos participantes da pesquisa era inferior a 18 anos.

Foram inclusas revisões sistemáticas atualizadas por centros de referências e trabalhos que forneciam informações para determinação da emetogenicidade da quimioterapia administrada, independente do protocolo utilizado.

Foram excluídos os estudos em que o tratamento oncológico tinha sido realizado em adultos e também aqueles em que as náuseas e os vômitos eram relacionados com patologias gastrointestinais, com radioterapia e com cirurgias ou pós-operatórios.

Baseando-se na necessidade de reunir informações sobre o problema de pesquisa, foram selecionados os descritores, os quais obtiveram 453 artigos, sendo este um número expressivo de publicações durante o período de 2015 a 2020, porém, após aplicação dos critérios metodológicos de inclusão e exclusão, foram selecionados 9 artigos conforme mostra a (Figura 1). 
Figura 1- Fluxograma de seleção dos artigos para a composição da revisão integrativa.

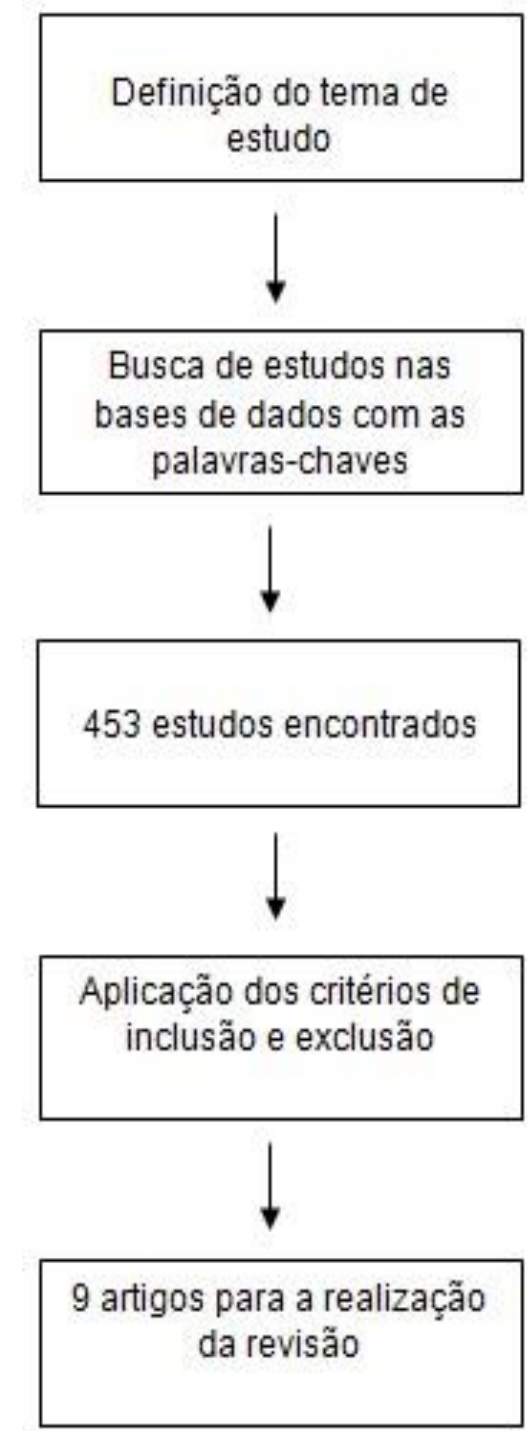

Fonte: LIMA IL, et al., 2021.

\section{RESULTADOS E DISCUSSÃO}

Após a busca nas bases de dados com as palavras-chaves, houve um total de 453 estudos encontrados. Houve a exclusão de resultados que apareceram mais de uma vez, dos que receberam apoio financeiro da indústria farmacêutica, dos que tratavam de intervenções psicoeducacionais ou daqueles em que os resumos/títulos não estavam compatíveis com os critérios de inclus ão. Após aplicação de todos os filtros de inclusão e exclusão, 9 artigos foram selecionados (Quadro 1). 
Quadro 1 - Caracterização dos estudos analisados.

\begin{tabular}{|c|c|c|}
\hline $\mathbf{N}$ & Autores (Ano) & $\begin{array}{l}\text { Principais achados } \\
\end{array}$ \\
\hline 1 & $\begin{array}{l}\text { CHAUDHARY NK, } \\
\text { et al. (2019) }\end{array}$ & $\begin{array}{l}\text { Ensaio clínico, não cego e randomizado. Compara a eficácia, segurança e } \\
\text { custo-efetividade da palonosetrona em relação à Ondansetrona. A } \\
\text { Palonosetrona mostrou resultados comparáveis a Ondansetrona, porém } \\
\text { apresentou vantagem econômica na sua utilização. }\end{array}$ \\
\hline 2 & $\begin{array}{l}\text { DUPUIS LL, et al. } \\
\qquad(2017)\end{array}$ & $\begin{array}{l}\text { Revisão sistemática. Atualiza as recomendações realizadas em } 2009 \text {, através } \\
\text { de estudos randomizados, para prevenção de êmese aguda induzida por } \\
\text { quimioterapia em crianças. Recomenda um antagonista 5-HT3 (granisetrona, } \\
\text { ondansetrona, palonosetrona ou tropisetrona) + Dexametasona + Aprepitanto } \\
\text { para crianças recebendo quimioterapia de alta ou moderada emetogenicidade. } \\
\text { Para quimioterapia de baixa emetogenicidade, um antagonista de 5-HT3 é } \\
\text { recomendado. }\end{array}$ \\
\hline 3 & $\begin{array}{l}\text { HESKETH PJ, et } \\
\text { al. (2017) }\end{array}$ & $\begin{array}{l}\text { Revisão sistemática. Atualização da diretriz da American Society of Clinical } \\
\text { Oncology (ASCO) sobre o uso de antieméticos em oncologia. Recomenda para } \\
\text { alto grau emetogênico: antagonista 5-HT3, Dexametasona e Aprepitanto. } \\
\text { Moderado: antagonista 5-HT3 e Dexametasona. Baixo: antagonista 5-HT3 }\end{array}$ \\
\hline 4 & $\begin{array}{l}\text { KANG HJ, et al. } \\
(2018)\end{array}$ & $\begin{array}{l}\text { Estudo randomizado, duplo-cego, controlado por comparador ativo, de grupo } \\
\text { paralelo, multicêntrico, estudo principal de Fase } 3 \text {. Verifica a resposta do uso do } \\
\text { Aprepitanto com Ondansetrona comparado com placebo e Ondansetrona em } \\
\text { crianças de entre } 6 \text { meses e } 17 \text { anos. O Aprepitanto foi superior para a } \\
\text { prevenção de náuseas e vômitos induzidos por quimioterapia do tipo tardia, } \\
\text { além de eficaz e seguro, com o uso durante } 3 \text { dias, em indivíduos pediátricos. }\end{array}$ \\
\hline 5 & $\begin{array}{l}\text { MORA J, et al. } \\
(2019)\end{array}$ & $\begin{array}{l}\text { Estudo de fase 2B, global, multicêntrico, randomizado, parcialmente cego. } \\
\text { Compara, utilizando dados de adultos, o perfil farmacológico, segurança e } \\
\text { tolerabilidade do Fosaprepitanto administrado com Ondansetrona, com/sem } \\
\text { Dexametasona em crianças de até } 17 \text { anos. } \\
\text { O Fosaprepitanto foi bem tolerado, necessitando de doses maiores para o } \\
\text { controle em pacientes menores de } 12 \text { anos. }\end{array}$ \\
\hline 6 & $\begin{array}{l}\text { OKUMURA LM, et } \\
\text { al. (2017) }\end{array}$ & $\begin{array}{l}\text { Revisão sistemática. Revisa a eficácia e segurança do Aprepitanto em } \\
\text { combinação com Ondansetrona e Dexametasona em crianças e adolescentes } \\
\text { em quimioterapia moderada a altamente emetogênica. A terapia tripla diminuiu } \\
\text { o risco de vômitos induzidos por quimioterapia, sem aumentar a ocorrência de } \\
\text { neutropenia febril. No entanto, estudos futuros devem se concentrar na } \\
\text { avaliação de fatores de risco para náuseas e vômitos, pois muitos pacientes não } \\
\text { obtiveram uma resposta antiemética completa. }\end{array}$ \\
\hline 7 & $\begin{array}{l}\text { PATEL P, et al. } \\
(2017)\end{array}$ & $\begin{array}{l}\text { Revisão sistemática. Atualiza as diretrizes de prática clínica de } 2013 \text { baseadas } \\
\text { em revisões sistemáticas. Recomenda para alto grau emetogênico: antagonista } \\
\text { 5-HT3, Dexametasona e Aprepitanto. Moderado: antagonista 5-HT3 e } \\
\text { Dexametasona. Na impossibilidade do uso da Dexametasona, recomenda a } \\
\text { Palonosetrona e Aprepitanto ou apenas a Palonosetrona para ambos os graus } \\
\text { emetogênicos. }\end{array}$ \\
\hline 8 & $\begin{array}{l}\text { POLITO S, et al. } \\
\qquad(2018)\end{array}$ & $\begin{array}{l}\text { Revisão multicêntrica retrospectiva. Descreve a segurança e eficácia da } \\
\text { Nabilona administrada a pacientes pediátricos para prevenir náuseas e vômitos } \\
\text { induzidos por quimioterapia. O controle agudo das náuseas e vômitos, da } \\
\text { nabilona como parte de seu regime antiemético foi pobre. Recomenda a } \\
\text { continuidade das pesquisas. }\end{array}$ \\
\hline 9 & $\begin{array}{c}\text { SING EPC, et al. } \\
(2019)\end{array}$ & $\begin{array}{l}\text { Revisão sistemática. Faz recomendações para a seleção apropriada da } \\
\text { profilaxia antiemética em pacientes oncológicos pediátricos. Recomenda para } \\
\text { alto grau emetogênico: antagonista 5-HT3, Dexametasona e Aprepitanto. } \\
\text { Moderado: antagonista 5-HT3 e Dexametasona ou a Palonosetrona. Baixo: } \\
\text { antagonista 5-HT3. Na ausência de dados pediátricos, utilizar dados dos } \\
\text { pacientes adultos. }\end{array}$ \\
\hline
\end{tabular}

Fonte: LIMA IL, et al., 2021.

Em relação aos medicamentos, houve um grande avanço no desenvolvimento de agentes antieméticos nos últimos vinte e cinco anos, porém os sintomas de náuseas e êmese, ainda são responsáveis por perdas significativas na qualidade de vida e redução da adesão ao tratamento (CHAUDHARY LM, et al., 2019; 
HESKETH PJ, et al., 2017; MORA J, et al., 2019; OKUMURA LM, et al., 2017; SANTOS SLF, et al., 2015; SING EPC, et al., 2019).

A terapêutica farmacológica para prevenir e controlar náuseas e êmese decorrentes do uso dos quimioterápicos é baseada na utilização de medicamentos antieméticos, que têm como primeiros representantes, os antagonistas de dopamina (metoclopramida, clorpromazina), substituídos devido os ef eitos extrapiramidais pelos antagonistas de serotonina (5-HT3) de primeira geração (ondansetrona e granisetrona) e os anti-histamínicos (dimenidrinato e prometazina), que caíram em desuso devido à pouca eficácia, passando a utilizar os corticosteroides dexametasona e metilprednisolona (SANTOS SLF, et al., 2015).

A prevenção deve acontecer já no primeiro ciclo de tratamento, a fim de diminuir a frequência desses ef eitos em ciclos posteriores, a possibilidade de internações hos pitalares, e consequente elevação dos gastos para as instituições (ALMEIDA RGL, et al., 2015; SANTOS SLF, et al., 2015).

Assim, é utilizada a classificação de emetogenicidade dos medicamentos que compõem a quimioterapia (alto grau emetogênico: $>90 \%$ de incidência de náuseas e êmese na ausência de profilaxia, moderado: 30 a $90 \%$, baixo: 10 a $30 \%$ e mínimo: $<10 \%$ ) uma vez que, os fatores tidos como de risco para pacientes adultos como: idade, raça e sexo, ainda não foram determinados de forma conclusiva para pacientes pediátricos. (ALMEIDA RGL, et al. 2015; DUPUIS LL, et al., 2017; HESKETH PJ, et al., 2017; MORA J, et al., 2019; PATEL P, et al., 2017; SANTOS SLF, et al., 2015; SING EPC, et al., 2019).

Observa-se a predominância da discussão e utilização da classe medicamentosa dos antagonistas de serotonina (5-HT3), que atuam através do bloqueio desses receptores no sistema nervoso central e periférico (principalmente no trato gastrintestinal) (CLORIDRATO DE ONDANSETRONA, 2021). São inúmeros estudos sobre a segurança da utilização na pediatria, que tem como maior representante, a Ondansetrona, ficando recomendada a utilização, anteriormente ao início do ciclo de quimioterapia, para evitar a êmese aguda (DUPUIS LL, et al., 2017; HESKETH PJ, et al., 2017; OKUMURA LM, et al., 2017; PATEL P, et al., 2017; SANTOS SLF, et al., 2015; SING EPC, et al., 2019).

Logo, apresenta resultados positivos para os diferentes graus emetogênicos dos medicamentos que compõem o tratamento quimioterápico, podendo ser utilizado sozinho, para baixo risco emético ou em combinação com outras classes medicamentosas, para os riscos emetogênicos moderado ou alto (DUPUIS et al., 2017; HESKETH PJ, et al., 2017; OKUMURA LM, et al., 2017; PATEL P, et al., 2017; SING EPC, et al., 2019).

Ainda da classe dos antagonistas de 5-HT3, vários estudos como Chaudhary NK, et al. (2019), Hesketh PJ, et al. (2017), Patel P, et al. (2017), Santos GLF et al. (2015), Sing EPC, et al. (2019), destacam a utilização da Palonosetrona, pois apresenta bons resultados de controle da êmese aguda ou tardia, mesmo quando utilizada sozinha, em razão do tempo de meia-vida longa (>40h), além de equivaler, segundo Sing EPC, et al. (2019), a um antagonista de 5-HT3 de primeira geração (Granisetrona ou Ondansetrona) adicionado da Dexametasona.

Os estudos de Okumura LM, et al. (2017), Kang HJ, et al. (2018) e Santos SLF, et al., (2015) apoiam a eficácia e a segurança do uso por via oral do antagonista de receptores da neurocinina1 (NK1) - Aprepitanto, que bloqueia a interação da substância $P$ e seus receptores neuronais, atuando na êmese aguda e tardia em crianças utilizado em regime de três dias em crianças de todas as faixas de idade, especialmente para prevenção das reações tardias.

Já no estudo de Okumura LM, et al. (2017), os autores destacam a necessidade de aprofundar as pesquisas determinando os fatores de risco na pediatria para a incidência de náuseas e êmese durante 0 tratamento quimioterápico, já que o conhecimento das características emetogênicas dos medicamentos não foram suficientes para o controle antiemético completo, mesmo utilizando a terapia tripla (Aprepitanto associado a antagonista do receptor 5-HT3 e corticosteroides).

A realidade encontrada durante essa pesquisa ainda é semelhante ao estudo de Okumura LM, et al. (2017), uma vez que ainda são utilizadas as características emetogênicas dos medicamentos para a def inição 
da terapia antiemética, devido a falta de estudo para a determinação dos fatores de risco relacionadas aos pacientes pediátricos. Observa-se ainda, a falta da classificação emética para vários medicamentos comumente utilizados no tratamento pediátrico, sendo necessária a utilização da classificação emética ou farmacocinética utilizada pelos pacientes adultos, conforme citado por Sing EPC, et al. (2019) e Mora J, et al. (2019).

Outro medicamento do grupo de antagonista de receptores da neurocinina1 (NK1) é o pró -fármaco do Aprepitanto, o Fosaprepitanto. Ambos de uso endovenoso é apresentado por Mora J, et al. (2019), como uma alternativa no tratamento, principalmente naqueles que não conseguem receber dosagem oral antiemética, bem tolerado e apresentando não inferioridade quando administrado em uma dose única de fosaprepitanto $(150 \mathrm{mg}$ ) comparado com o regime padrão do aprepitanto em 3 dias. Porém, ainda são poucos os trabalhos que exploram tal medicamento na pediatria, pois foi a única publicação selecionada durante a realização desta pesquisa.

Os derivados de Canabinóides que são aprovados pela Food and Drug Administration (FDA) dos Estados Unidos são o Dronabinol (apenas para adultos) e Nabilona, apresentado por Hesketh PJ, et al. (2017), não havendo, até aquele momento, estudos sobre o uso na pediatria. No trabalho, Barros e Prado (2015), direcionado à pediatria, fala da limitação para o uso desses medicamentos devido aos efeitos adversos provocados, como: tontura, sonolência e alteração de humor, assim como o exposto por Polito S, et al. (2018). Nesse estudo, ocorre o questionamento da contribuição da Nabilona, uma vez que mesmo tendo seu uso associado a um antagonista de 5 -HT3, a proporção de crianças que obtiveram o controle agudo completo das êmeses foi baixo.

Com isso, o que se observa com a análise dos trabalhos citados anteriormente, é que são várias as classes medicamentosas aplicáveis ou ainda em aprofundamento sobre sua utilização para o controle de náuseas e êmeses induzidas pela quimioterapia na pediatria. Tais estudos estão baseados nos diferentes graus emetogênicos dos medicamentos, chegando as seguintes terapias exposto no Quadro 2.

Quadro 2 - Potencial emetogênico dos quimioterápicos e os medicamentos indicados para prevenção e controle de náuseas e êmese.

\begin{tabular}{|c|c|c|c|c|}
\hline $\begin{array}{c}\text { Potencial } \\
\text { Emetogênico } \\
\text { da } \\
\text { Quimioterapia }\end{array}$ & $\begin{array}{c}\text { Menores de 6 } \\
\text { meses e o uso da } \\
\text { Dexametasona } \\
\text { permitido }\end{array}$ & $\begin{array}{c}\text { Menores de 6 } \\
\text { meses e o uso da } \\
\text { Dexametasona } \\
\text { não for permitido }\end{array}$ & Maiores de 6 Meses & $\begin{array}{c}\text { Maiores de 6 } \\
\text { Meses e o uso da } \\
\text { Dexametasona } \\
\text { não for permitido }\end{array}$ \\
\hline Alto & $\begin{array}{c}\text { Antagonista de 5- } \\
\text { HT3 (Granisetrona, } \\
\text { Ondansetrona ou } \\
\text { Palonosetrona), } \\
\text { Dexametasona }\end{array}$ & Palonosetrona & $\begin{array}{c}\text { Antagonista de 5- } \\
\text { HT3 (Granisetrona, } \\
\text { Ondansetrona ou } \\
\text { Palonosetrona), } \\
\text { Dexametasona e } \\
\text { Aprepitanto }\end{array}$ & $\begin{array}{c}\text { Palonosetrona e o } \\
\text { Aprepitanto }\end{array}$ \\
\hline Moderado & $\begin{array}{c}\text { Antagonista de 5- } \\
\text { HT3 (Granisetrona } \\
\text { ou Ondansetrona) e } \\
\text { Dexametasona }\end{array}$ & Palonosetrona & $\begin{array}{c}\text { Antagonista de 5-HT3 (Granisetrona, } \\
\text { Ondansetrona ou Palonosetrona) e o } \\
\text { Aprepitanto }\end{array}$ \\
\hline Baixo & \multicolumn{2}{|c|}{ Antagonista de 5-HT3 (Granisetrona ou Ondansetrona) } \\
\hline Mínimo & \multicolumn{3}{|c|}{ Não é necessária terapia profilática antiemética } \\
\hline
\end{tabular}

Fonte: LIMA IL, et al., 2021.

Para os medicamentos com alto risco emético, Dupuis LL, et al. (2017), Hesketh PJ, et al. (2017), Patel P, et al. (2017) e Sing EPC, et al. (2019), recomendam a terapia tripla: um antagonista de 5-HT3 (Granisetrona, Ondansetrona ou Palonosetrona), Dexametasona e Aprepitanto para crianças maiores de 6 meses de idade.

No trabalho de Santos SLF, et al., (2015) a Dexametasona é recomendada por atuar inibindo a síntese de prostaglandinas e evitar a estimulação e ativação do centro do vômito, porém, para Dupuis LL, et al. (2017), Hesketh PJ, et al. (2017), Patel P, et al. (2017) e Sing EPC, et al. (2019) a Dexametasona deve ser utilizada 
de maneira criteriosa após avaliação das condições clinicas e identificação do tipo de câncer. Não há, portanto, possibilidade de piora clínica pelo o uso de corticosteroide incluso na terapia antiemética. Já a inclusão do Aprepitanto na terapia antiemética deve ser precedida da verificação de possíveis interações com o protocolo quimioterápico prescrito.

Na impossibilidade de receber o Aprepitanto, Dupuis LL, et al. (2017), Hesketh PJ, et al. (2017), Patel P, et al. (2017) e Sing EPC, et al. (2019) recomendam um antagonista de 5-HT3 (Granisetrona, Ondansetrona ou Palonosetrona) e Dexametasona. Se não for possível o uso da Dexametasona, Hesketh PJ, et al. (2017) e Patel P, et al. (2017), recomendam a Palonosetrona e o Aprepitanto. Quando não for possível o uso de Aprepitanto, Patel P, et al. (2017) e Sing EPC, et al. (2019), recomendam somente a Palonosetrona.

Para Dupuis LL, et al. (2017), Hesketh PJ, et al. (2017), Patel P, et al. (2017) e Sing EPC, et al. (2019) o recomendado para os pacientes em uso de medicamentos com potencial emético moderado, é um antagonista de 5-HT3 (Granisetrona, Ondansetrona ou Palonosetrona) e Dexametasona. Na impossibilidade do uso da Dexametasona, os autores citados acima, indicam um antagonista de 5-HT3 (Granisetrona, Ondansetrona ou Palonosetrona) e o Aprepitanto ou apenas a Palonosetrona, na impossibilidade do Aprepitanto.

Quando a terapia farmacológica utilizada for compostapor medicamentos com baixo risco emético, Dupuis LL, et al. (2017) e Hesketh PJ, et al. (2017) recomendam apenas um antagonista de 5-HT3 (Granisetrona ou Ondansetrona) e para os medicamentos com risco emético mínimo Hesketh PJ, et al. (2017) afirmam que não se faz necessário utilizar a terapia profilática antiemética, assim como Dupuis LL, et al. (2017), que partilha da mesma ideia, porém baseando-se em estudos realizados com adultos, já que não foram encontrados pesquisas com crianças para essa faixa emética mínima.

Todas essas informações formam um compilado de estudos clínicos relacionados à segurança farmacológica de tais medicamentos e sua aplicabilidade na pediatria, para formular o Quadro 2, citado anteriormente, para o controle de náuseas e êmese específico para essa faixa etária, não levando em consideração os valores de mercado para a seleção deles.

Interessante observar o posicionamento Hesketh PJ, et al. (2017) e Patel P, et al. (2017), quanto à impossibilidade/dificuldade do uso da Palonosetrona e principalmente do Aprepitanto, destacando não somente as características farmacológicas, a aplicabilidade na pediatria ou a necessidade da realização de novos estudos, mas justamente o impacto financeiro, que influencia na sua escolha e utilização pelas instituições e pacientes, já que para serem implementados como recomendações devem levar em consideração a realidade financeira para serem factíveis.

No Brasil o cenário de altos valores para aquisição desses medicamentos não é diferente, já que a ampola de Palonosetrona pode chegar a $\mathrm{R} \$ 676,00$ (seiscentos e setenta e seis reais), referente a um dia de sessão de quimioterapia ou a caixa com 3 (três) comprimidos de Aprepitanto, que corresponde a uma semana de tratamento quimioterápico, pode custar $\mathrm{R} \$ 867,00$ (oitocentos e sessenta e sete reais), o que torna esses medicamentos inviáveis e inacessíveis para a maioria da população (MINISTÉRIO DA SAÚDE, 2019).

A regulamentação do preço desses e de todos os medicamentos comercializados no Brasil é realizada pela Câmara de Regulação do Mercado de Medicamentos (CMED), que monitora a comercialização, atualiza e disponibiliza os preços para consulta mensalmente, aplica penalidades quando suas regras são descumpridas, é fixa e monitora a aplicação do desconto mínimo obrigatório para compras públicas (MINISTÉRIO DA SAÚDE, 2019).

Outra dificuldade relacionada aos custos aqui no Brasil é o fato de tais medicamentos não estarem na Classificação farmacológica da Secretaria Municipal de Belém (SESMA) compondo a Relação Municipal de Medicamentos (REMUME) Belém (2018) e na Relação Nacional de Medicamentos Essenciais (REMANE) que abrange a seleção e a padronização dos medicamentos utilizados no âmbito do Sistema Único de Saúde (SUS) conforme Ministério da Saúde (2019), restringindo seu uso. Esse que poderia ser empregado para melhorar as evidências científicas disponíveis sobre a sua utilização, não só no Brasil, como na América Latina, já que não foram encontrados estudos aplicados nessa região durante a pesquisa. 
Assim, com o farmacêutico oncológico garantindo a aplicação e o uso correto da terapia antiemética, principalmente no início das sessões de quimioterapia, além de evitar as reações agudas e/ou tardias, evita as reações antecipatórias em ciclos futuros, devido ao controle ineficaz em ciclos anteriores, contribuindo na melhoria da qualidade de vida, evitando novas internações resultantes do agravamento dessas reações adversas como a desnutrição, anorexia, desidratação e complicações emocionais como ansiedade e estresse. (ALMEIDA RGL, et al., 2015; SANTOS SLF, et al., 2015).

\section{CONSIDERAÇÕES FINAIS}

Na construção desta revisão integrativa, observa-se a importância da temática abordada, estando no foco de centros de referência na Europa e América do Norte, os quais recomendam a utilização dos medicamentos antieméticos através de protocolos, utilizando classes medicamentosas seguras e eficientes para essa faixa etária como: os antagonistas de 5-HT3, corticoide e o antagonista da neurocinina-1. Porém, ainda são poucos os estudos fora desses centros de referência, como no Brasil e América Latina, onde não foram encontrados estudos aplicados a essa população. Diante disso, é importante trazer e aplicar essa informação na nossa realidade, visando à melhoria da assistência prestada ao paciente.

\section{REFERÊNCIAS}

1. ALMEIDA RGL, et al. O Manejo da Êmese em uma Unidade Oncológica: a necessidade da intervenção farmacêutica em tempo real. Revista Brasileira de Cancerologia, 2015;61(2):115-21.

2. BELÉM. Secretaria Municipal de Saúde (SESMA). Classificação farmacológica medicamentos para dispensação nas unidades de conforme local de acesso. 2018. Disponível em: https://sistemas.belem.pa.gov.br/diario/painel. Acessado em: 15 de janeiro de 2020.

3. CHAUDHARY NK, et al. Palonosetron is a Better Choice Compared with Ondansetron for the Prevention of Chemotherapy-induced Nausea and Vomiting in a Resource-limited Pediatric Oncology Center: Results From a Randomized Control Trial. Journal of pediatric hematology/oncology, 2019;41(4):294-297.

4. CLORIDRATO DE ONDANSETRONA. Solução injetável $2 \mathrm{mg} / \mathrm{mL}$. Farmacêutica Responsável: Cristal Mel Guerra e Silva. Ribeirão das Neves: Hypofarma-Instituto de Hypodermia e Farmácia Ltda. Disponível em: http://hypofarma.com.br/site/wp-content/uploads/2021/07/Bula_Cloridrato_de_Ondansetrona_Profissional.pdf. Acessado em:25 de janeiro de 2020.

5. CONSELHO FEDERAL DE FARMÁCIA (CRF). Serviços farmacêuticos diretamente destinados ao paciente, à família e à comunidade: contextualização e arcabouço conceitual. 2016. Disponível em: https://www.cff.org.br/userfiles/Profar_Arcabouco_TELA_FINAL.pdf. Acessado em:25 de janeiro de 2020.

6. DUPUIS LL, et al. 2016 updated MASCC/ESMO consensus recommendations: Prevention of acute chemotherapyinduced nausea and vomiting in children. Support Care Cancer, 2017;25(1) 323-331.

7. HESKETH PJ, et al. Antiemetics: American Society of Clinical Oncology clinical practice guideline update. Journal of Clinical Oncology, 2017;35(28):3240-3261.

8. INSTITUTO NACIONAL DE CÂNCER JOSÉ ALENCAR GOMES DA SILVA (INCA). Coordenação de Prevenção e Vigilância. Incidência, mortalidade e morbidade hospitalar por câncer em crianças, adolescentes e adultos jovens no Brasil: informações dos registros de câncer e do sistema de mortalidade. 2016. Disponível em: https://www.inca.gov.br/sites/ufu.sti.inca.local/files//media/document/incidencia-mortalidade-morbidade-hospitalarpor-cancer.pdf. Acessado em: 13 de maio de 2020.

9. INSTITUTO NACIONAL DE CÂNCER JOSÉ ALENCAR GOMES DA SILVA (INCA). Estimativa 2020:incidência de $\begin{array}{lllll}\text { câncer no } & \text { Brasil. } & 2019 . & \text { Disponível }\end{array}$ https://www.inca.gov.br/sites/ufu.sti.inca.local/files//media/document/estimativa-2020-incidencia-de-cancer-nobrasil.pdf. Acessado em: 6 de agosto de 2020.

10. KANG HJ, et al. Aprepitant for the prevention of chemotherapy-induced nausea and vomiting in paediatric subjects: An analysis by age group. Pediatric Blood and Cancer, 2018;65(10):1-8.

11. MINISTÉRIO DA SAÚDE. Agência Nacional de Vigilância Sanitária (Anvisa). Câmara de Regulação do Mercado de Medicamentos. Secretaria Executiva. Preços máximos de medicamentos por princípio ativo. 2021. Disponível em: http://antigo.anvisa.gov.br/documents/374947/6048620/LISTA_CONFORMIDADE_2020_10_v1.pdf/7b88a38f-1b2f4768-b589-f62b4beb1762. Acessado em: 25 de janeiro de 2020.

12. MINISTÉRIO DA SAÚDE. Secretaria de Atenção à Saúde. Departamento de Atenção Especializada e Temática. Protocolo de diagnóstico precoce para oncologia pediátrica. 2017 . Disponível em: https://bvsms.saude.gov.br/bvs/publicacoes/protocolo_diagnostico. Acesso em:25 de janeiro de 2020.

13. MINISTÉRIO DA SAÚDE. Secretaria de Atenção à Saúde. Departamento de Regulação, Avaliação e Controle Coordenação Geral de Sistemas de Informação. Manual de bases técnicas da oncologia - SIA/SUS - sistema de informações ambulatoriais. 2019. Disponivel em: https://www.inca.gov.br/sites/ufu.sti.inca.local/files/media/docume $\mathrm{nt} / \mathrm{m}$ anual-oncologia-26a-edicao.pdf. Acessado em: 15 de agosto de 2020. 
14. MINISTÉRIO DA SAÚDE. Secretaria de Ciência, Tecnologia, Inovação e Insumos Estratégicos em Saúde. Departamento de Assistência Farmacêutica e Insumos Estratégicos. Relação Nacional de Medicamentos Essenciais 2020 (RENAME). 2019. Disponível:https://bvsms.saude.gov.br/bvs/publicacoes/relacao_medicamento S_rename_2020.pdf. Acessado em:25 de janeiro de 2020.

15. MORA J, et al. Pharmacokinetics/pharmacodynamics, safety, and tolerability of fosaprepitant for the prevention of chemotherapy-induced nausea and vomiting in pediatric cancer patients. Pediatric Blood and Cancer, 2019;66(6): $1-11$.

16. MUTTI CF, et al. Perfil Clínico-epidemiológico de Crianças e Adolescentes com Câncer em um Serviço de Oncologia. Revista Brasileira de Cancerologia, 2018;64(3):293-300.

17. OKUMURA LM, et al. Aprepitant in pediatric patients using moderate and highly emetogenic protocols: a systematic review and meta-analyses of randomized controlled trials. British Journal of Clinical Pharmacology, 2017;83:1108 1117.

18. PATEL $P$, et al. Guideline for the prevention of acute chemotherapy-induced nausea and vomiting in pediatric cancer patients: A focused update. Pediatric Blood and Cancer, 2017;64(10):1-12.

19. POLITO S, et al. Safety and efficacy of nabilone for acute chemotherapy induced vomiting prophylaxis in pediatric patients: A multicenter, retrospective review. Pediatric Blood and Cancer, 2018; 1-5. e27374.

20. QUEIROGA HM, et al. Avaliação de náusea e êmese em pacientes sob quimioterapia em uma Unidade de Alta Complexidade Saúde de Vitória da Conquista/BA. Revista Brasileira de Pesquisa em Saúde, 2017;19:126-132.

21. SANTOS SLF, et al. Utilização de medicamentos antieméticos em oncologia pediátrica: riscos e benefícios. Revista Brasileira de Pesquisa em Saúde, 2015; 7(3): 141 -150.

22. SING EPC, et al. Classification of the acute emetogenicity of chemotherapy in pediatric patients: A clinical practice guideline. Pediatric Blood and Cancer, 2019;66(5): 1-7. 